\title{
Aberrant Frontoparietal Function during Recognition Memory in Schizophrenia: A Multimodal Neuroimaging Investigation
}

\author{
Anthony P. Weiss, ${ }^{1,2}$ Cameron B. Ellis, ${ }^{1,2}$ Joshua L. Roffman, ${ }^{1,2}$ Steven Stufflebeam, ${ }^{2}$ Matti S. Hamalainen, ${ }^{2}$ \\ Margaret Duff, ${ }^{1,2}$ Donald C. Goff, ${ }^{1 \star}$ and Daniel L. Schacter ${ }^{3 \star}$ \\ ${ }^{1}$ Department of Psychiatry, Massachusetts General Hospital, Boston, Massachusetts 02114, ${ }^{2}$ Athinoula A. Martinos Center for Biomedical Imaging, \\ Charlestown, Massachusetts 02129, and ${ }^{3}$ Department of Psychology, Harvard University, Cambridge, Massachusetts 02138
}

\begin{abstract}
Prefrontal-parietal networks are essential to many cognitive processes, including the ability to differentiate new from previously presented items. As patients with schizophrenia exhibit structural abnormalities in these areas along with well documented decrements in recognition memory, we hypothesized that these patients would demonstrate memory-related abnormalities in prefrontal and parietal physiology as measured by both functional magnetic resonance imaging and magnetoencephalography (MEG). Medicated outpatients with schizophrenia $(n=18)$ and age-matched healthy control subjects $(n=18)$ performed an old-new recognition memory task while physiological data were obtained. Whereas controls exhibited strong, bilateral activation of prefrontal and posterior parietal regions during successful identification of old versus new items, patients exhibited greatly attenuated activation of the right prefrontal and parietal cortices. However, within the patient group, there was strong correlation between memory performance and activation of these right-sided regions as well as a tight correlation between old-new effect-related activations in frontal and parietal regions, a pattern not seen in control subjects. Using MEG, control subjects - but not patients - exhibited a sequential pattern of old $>$ new activity in the left posterior parietal cortex and then right prefrontal cortex; however, patients uniquely exhibited old $>$ new activity in right temporal cortex. Collectively, these findings point to markedly different distributions of regional specialization necessary to complete the old-new item recognition task in patients versus controls. Inefficient utilization of prefrontal-parietal networks, with compensatory activation in temporal regions, may thus contribute to deficient old-new item recognition in schizophrenia.
\end{abstract}

\section{Introduction}

Patients with schizophrenia exhibit deficits in memory that limit their functional capacity within the community (Green, 2006) and interfere with their ability to adhere to treatment (Jeste et al., 2003; Heinrichs et al., 2008). These deficits are present from the earliest stages of the illness and cannot be fully explained as a side effect of pharmacotherapy (Simon et al., 2007). As currently available treatments have relatively little effect on these cognitive deficits (Keefe et al., 2007), identifying their underlying neural causes remains an important area for scientific inquiry.

The importance of the prefrontal cortex in normal human memory, and its potential role in the memory deficits seen in patients with schizophrenia, have been well described (Barbas and Zikopoulos, 2007; Ragland et al., 2007; Tan et al., 2007b;

Received Feb. 5, 2009; revised June 23, 2009; accepted June 30, 2009

This work was supported by National Institutes of Health (NIH) Grant K23-MH06019 (A.P.W.), NIH Grant R01MH060941 (D.L.S.), Howard Hughes Medical Institute Physician-Scientist Early Career Award (J.L.R.), the MIND Institute, and National Center for Research Resources Regional Resources Grant P41RR14075 (Martinos Center). We thank Tali Ditman and Michael Zussman for assistance with the paradigm development, and Dr. Lindsay Jubelt, Deidre von Pechmann, and Kaila Norman for assistance with data acquisition.

${ }^{*} D$.C.G. and D.L.S. contributed equally to this work.

Correspondence should be addressed to Dr. Anthony P. Weiss, Department of Psychiatry, Massachusetts General Hospital, 1 Bowdoin Square, Boston, MA 02114.E-mail: aweiss@partners.org.

D0I:10.1523/JNEUROSCI.0617-09.2009

Copyright $\odot 2009$ Society for Neuroscience ～0270-6474/09/2911347-13\$15.00/0
Ranganath et al., 2008). Recent evidence has also highlighted an important contributory role of the parietal cortices in normal declarative memory processes (Wagner et al., 2005; Cabeza et al., 2008). This is not necessarily surprising; as the principal posterior heteromodal cortex, the parietal lobes represent a critical information crossroad, bringing together information experienced through diverse sensory streams. The importance of the parietal lobe in the pathophysiology of schizophrenia is also being increasingly recognized, with at least 10 studies demonstrating structural abnormalities specifically within the inferior parietal lobule (for review, see Torrey, 2007). Despite these findings, there has been as yet relatively little research on the functional role of this region in memory processing in patients with schizophrenia.

The connection between parietal and prefrontal cortices is thought to be particularly important and forms the basis for network-based models of human cognitive function (including episodic memory) (Fuster, 2006). The relevance of prefrontal and parietal activation to episodic memory have been robustly demonstrated in neurophysiology studies of the so-called "old-new effect" (Rugg and Curran, 2007), in which successful recognition of previously experienced items (relative to novel items) produces both characteristic event-related potentials (ERPs) in the posterior parietal and prefrontal cortices, as well as blood oxygenation level-dependent (BOLD) activation in the same regions (Wagner et al., 2005). We recently described significant perfor- 
mance deficits in schizophrenia patients during an old-new item recognition paradigm (Weiss et al., 2008). The current experiment sought to assess prefrontal and parietal physiology during the old-new task in the same group of patients and controls in light of these performance differences.

Prefrontal and parietal physiology was assessed using two complementary methods: functional magnetic resonance imaging (fMRI) and magnetoencephalography (MEG). The use of MEG allowed us to obtain a better "real-time" measure of the temporal activity within these regions, as well as complementing a blood-flow based analysis with one based directly on electromagnetic activity change. Based on the extant literature, we hypothesized that both the parietal and frontal components of this old-new effect would be diminished in patients with schizophrenia. This work was presented in part at the International Congress on Schizophrenia Research, 2007 and 2009 (Weiss et al., 2007; Roffman et al., 2009).

\section{Materials and Methods}

Subjects. The demographic characteristics of the enrolled subjects and a detailed description of the memory paradigm have been previously reported (Weiss et al., 2008) and will be briefly summarized below. Before enrollment of subjects, the protocol was approved by the institutional review boards of Partners HealthCare and the Commonwealth of Massachusetts Department of Mental Health. All participants provided written informed consent after a complete description of the study and administration of a brief questionnaire to ensure capacity to consent.

Eighteen outpatients (12 males and 6 females) with Diagnostic and Statistical Manual of Mental Disorders, Fourth Edition (DSM-IV)-defined schizophrenia based on SCID (Stuctured Clinical Interview for DSM-IV Axis I Disorders) criteria (First et al., 1995) and 18 age-matched control subjects ( 11 males and 7 females) participated in the study. Patients had a mean duration of illness of $15.6 \pm 11.4$ years, and all but one were taking a stable dose of antipsychotic medication (14 on secondgeneration antipsychotics, two on conventional antipsychotics, one on a combination of second-generation and conventional, and one unmedicated). Patients predominantly exhibited negative symptoms [mean Scale for the Assessment of Positive Symptoms (SAPS) (Andreasen, 1984) score, 16.2 \pm 16.0 ; Scale for the Assessment of Negative Symptoms (SANS) (Andreasen, 1983) score, $34.9 \pm 21.0$ ]. Control subjects were free of any axis I psychiatric condition and were not taking psychotropic medication. Neither patients nor control subjects had a history of major medical or neurological illness. No subject met DSM-IV criteria for alcohol or other substance use disorder (excepting nicotine dependence) within the past 3 months.

As described previously (Weiss et al., 2008), there were no significant between-group differences in age, parental socioeconomic status, or parental education. When compared with the patients with schizophrenia, control subjects had a higher level of attained formal education, better socioeconomic status, and higher overall verbal IQ as estimated by the North American Adult Reading Test (Blair and Spreen, 1989).

Old-new item recognition task. The experimental paradigm was adapted from a previously published source monitoring experiment (Wilding, 1999). Briefly, this recognition memory task comprised six alternating encoding and testing sessions conducted while subjects were positioned in the magnetic resonance imaging (MRI) and MEG scanners.

For fMRI scans, during each encoding session, participants saw and heard 26 consecutive words ( 13 spoken by a male; 13 spoken by a female) and identified the gender of the recorded voice using a keypad button. Words were rear-projected for $3000 \mathrm{~ms}$ onto a hemicircular tangent screen and viewed through a mirror mounted on the head coil of the magnetic resonance (MR) scanner. Each word was simultaneously presented aurally via pneumatic headphones at a clearly audible level using Presentation, version 0.80 (Neurobehavioral Systems). Immediately after this encoding session, participants were presented with 52 consecutive words ( 26 previously studied and 26 new) and were asked to identify
Table 1. Episodic memory performance under fMRI and MEG scanning conditions in patients and controls

\begin{tabular}{llll}
\hline & Controls (\%) & Patients (\%) & Statistics \\
\hline fMRI & & & \\
$\quad$ Corrected recognition rate & $70.4 \pm 19.9$ & $55.4 \pm 22.5$ & $t=2.12, \mathrm{df}=34, p<0.05$ \\
$\quad$ Source accuracy & $77.5 \pm 14.1$ & $73.3 \pm 16.0$ & $t=0.83, \mathrm{df}=34, p=0.41$ \\
MEG & & & \\
$\quad$ Corrected recognition rate & $74.1 \pm 19.0$ & $49.4 \pm 23.5$ & $t=3.43, \mathrm{df}=29, p<0.005$ \\
$\quad$ Source accuracy & $79.3 \pm 14.0$ & $72.2 \pm 11.9$ & $t=1.70, \mathrm{df}=29, p=0.10$ \\
\hline
\end{tabular}

Patients made significantly more errors differentiating new from previously presented items than did controls (as indicated by the corrected recognition rate, which corresponds to hit rate minus false alarm rate). However, there were no between-group differences in the source accuracy of items that were deemed to be old (i.e. correctly recalling that the word was originally presented in a male vs female voice).

the original source of the voice (i.e., male, female, or new). Words were presented one at a time visually for $3000 \mathrm{~ms}$ (plus a $500 \mathrm{~ms}$ interstimulus interval), with subjects indicating their response (male, female, or new) by pressing one of three buttons on a keypad.

For MEG scans, a nearly identical procedure was used, with the following modifications: (1) a counterbalancing scheme was developed such that the words used for the two visits (fMRI and MEG) were completely nonoverlapping; and (2) a series of $2 \mathrm{~s}$ "blink trials" were interposed into both the encoding and retrieval aspects of the MEG version of the paradigm. Subjects were encouraged to blink only during these trials, which occurred every $15.5 \mathrm{~s}$ and were later removed during routine preprocessing of the data. During encoding, visual stimuli were backprojected at eye level for $3000 \mathrm{~ms}$ onto a screen located 36 inches in front of the subject, and concurrently presented auditory stimuli were presented via pneumatic headphones as described above. The order of scans (i.e., having MEG vs fMRI first) was counterbalanced across subjects. The median time between the two scans was $15 \mathrm{~d}$ in both patients and controls.

Task performance analysis. Statistical analysis of the behavioral data was performed using SPSS, version 11.0 (SPSS). Standard measures of old-new recognition memory and source memory performance were calculated, with group means for these variables compared using an unpaired Student $t$ test. The corrected recognition rate (also sometimes called $\mathrm{Pr}$ ) is a standard measure of recognition memory accuracy. It is similar to the $d^{\prime}$ statistic from signal detection theory in that it attempts to control for response bias by including both "hits" and "false alarms."

MRI acquisition. Magnetic resonance imaging data were acquired with a 1.5 tesla Siemens Avanto whole-body clinical scanner (Siemens Medical Systems). After automated localizer, scout, and shimming procedures, a T1-weighted structural image with the same slice parameters as the functional images was obtained to aid in registration of functional to structural images. Functional images were then collected using a standard echoplanar imaging sequence [repetition time (TR), $2500 \mathrm{~ms}$; echo time (TE), $40 \mathrm{~ms}$; flip, $90^{\circ}$; voxel size, $3.1 \times 3.1 \times 5 \mathrm{~mm}$ ]. Thirty interleaved oblique coronal slices ( $5 \mathrm{~mm}$ width with $1 \mathrm{~mm}$ gap), oriented perpendicular to the anterior commissure-posterior commissure line, were obtained during each TR. In each of the six encoding runs, 37 images were collected (103 s scan duration), and in each of the six test runs 73 images were collected (193 s scan duration). After the functional runs were completed, two high-resolution structural scans were acquired using a threedimensional magnetization-prepared rapid-acquisition gradient echo (MPRAGE) sequence (TR, $2730 \mathrm{~ms}$; TE, $3.31 \mathrm{~ms}$; flip, $7^{\circ}$; voxel size, $1.3 \times$ $1 \times 1.3 \mathrm{~mm})$.

MRI analysis. The functional MRI data were analyzed using the FreeSurfer Functional Analysis Stream (FS-FAST) (http://surfer.nmr.mgh. harvard.edu/) (Dale et al., 1999; Fischl et al., 1999a,b). For each subject, functional scans were motion corrected (Cox, 1996), spatially smoothed (using a $6 \mathrm{~mm}$ full width at half-maximum Gaussian kernel), and intensity normalized. Based on visual inspection of the data, we found that distortions, ghosting, and other artifacts were present when the signalto-noise ratio (SNR) of the image was $<300$. This value was therefore used as a standard cutoff, with all runs with an SNR of $<300$ discarded from additional analyses. This led to the removal of 16 of 108 retrieval runs (14.8\%) from the control group and 25 of 105 retrieval runs $(23.8 \%)$ 


\section{Old vs New}
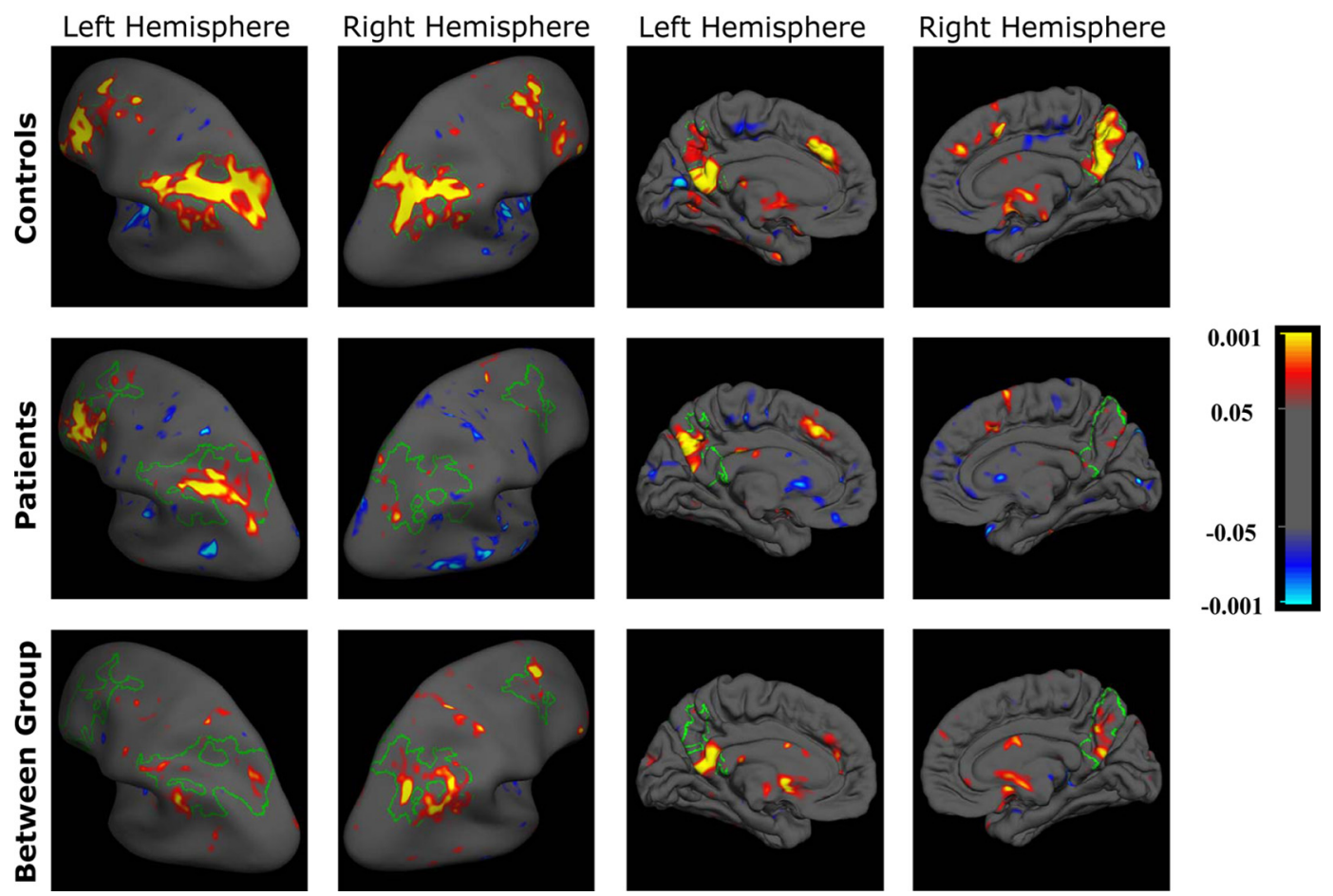

Figure 1. Statistical significance maps showing within- and between-group activation differences on the lateral (left side of figure) and medial (right side of figure) surface of the cortex. The "old versus new" contrast shows the activation differences between correctly recognized old items (regardless of source accuracy) and correctly rejected new items. Statistical significance is illustrated by color intensity, which represents an uncorrected vertex-level $p$ value. Significant clusters (based on cluster-level thresholding) within the control old-new contrast are outlined in green; these regions were used as functional regions of interest in subsequent analyses.

from the schizophrenia group. The data from remaining runs had a high mean SNR in each group (control, $621 \pm 218$; schizophrenia, $732 \pm 233$; between-group unpaired $t$ test, $t=1.48, \mathrm{df}=32, p=0.15$ ). To further evaluate whether differences in head motion could have contributed to between-group differences, we examined the mean motion vector (MMV) per TR in patients and controls. There was no difference between groups (control MMV, $0.042 \mathrm{~mm} / \mathrm{TR}$; patient MMV, $0.055 \mathrm{~mm} / \mathrm{TR} ; t=$ $-1.29, \mathrm{df}=32, p=0.21$ ).

Based on the item type and subject response, five event types were identified: accurate source recollection of an old item [source hit (SH)], inaccurate source recollection of an old item [source miss (SM)], no recollection of an old item [miss $(\mathrm{M})]$, accurate rejection of a new item [correct rejection $(\mathrm{CR})]$, and falsely labeling a new item as previously presented [false alarm (FA)]. The time window used for event estimation was composed of a multiple of the TR, extending from $5 \mathrm{~s}$ prestimulus to $40 \mathrm{~s}$ after stimulus. The data were detrended using a second-order polynomial to remove low-frequency scanner drift. Temporal correlations of the noise were estimated and corrected using a prewhitening function that takes the global residual noise into account.

Functional and anatomical volumes were aligned by registering the anatomical volumes to a $\mathrm{T} 1$ template, registering the first image of the first functional run to a $\mathrm{T}^{*}$ template, and then concatenating the registrations. Estimates of the hemodynamic response (HDR) for each of the five above-mentioned events were made by convolving the functional signal for each event with an assumed canonical HDR function. Individually averaged functional data were then resampled from native to spherical space for surface-based analysis, with subsequent application of a 20 iteration smoothing function. This level of smoothing is approximately equal to the surface-based application of a $6.6 \mathrm{~mm}$ full width at halfmaximum smoothing kernel.

Three contrasts were performed at the individual subject level: the difference in BOLD signal change between correctly identified old and new items (i.e., SH plus SM vs CR), the difference in BOLD signal change between correctly identified and forgotten old items (i.e., SH plus SM vs
$\mathrm{M})$, and the difference in BOLD signal change between correct and incorrect source identification (i.e., $\mathrm{SH}$ vs $\mathrm{SM}$ ). Subjects with a total event bin size of $<10$ items were excluded from those contrasts that included that event $(N=1$ control and 1 patient from the old vs new contrast; $N=$ 5 controls and 3 patients from the old vs miss contrast; and $N=4$ controls and 5 patients from the source identification contrast).

Within-group and between-group comparisons were generated for each contrast, with statistical significance based on a cluster-wise probability (CWP) threshold of 0.05 , a standard approach for correcting for multiple comparisons in surface-based analyses (Hagler et al., 2006) This CWP approach was implemented within FreeSurfer by initially running 1000 Monte Carlo simulations of synthesized white Gaussian noise using the smoothing, resampling, and averaging parameters of the functional analyses. This allowed us to determine the likelihood that a cluster of a certain size would be found by chance for a given vertex-based threshold.

In addition to this standard surface-based analysis, a secondary region of interest (ROI)-based approach was used, using the three bilateral regions found to be significant in the old versus new group-level contrast in control subjects (dorsolateral prefrontal cortex, posterior parietal cortex, and medial parietal cortex/precuneus) (Cannistraro et al., 2004). A functional label generated at the group level was morphed from an average subject to each individual subject (Fischl et al., 1999a,b), and the percentage signal change, averaged across the entire region, relative to a baseline offset, was obtained for all event types (Fischl et al., 2004). These values were incorporated into a mixed-model repeated-measures ANOVA, which examined the main effects of three within-subject measures (hemisphere, region, condition), and one between-subject measure (diagnosis), as well as their interactions. In addition, these ROI data were used in post hoc manner to explore the frontal-parietal activity relationship as well as the relationship between activity and performance using Pearson's correlation coefficient (two-tailed $\alpha$ of 0.05 ). Of note, by including data from the entirety of each individual's functionally defined ROI, as opposed to using the single most strongly activated vertex, we 
attempted to minimize the possibility of spurious correlations (Vul et al., 2009).

MEG acquisition. MEG data were acquired in a magnetically shielded room using a dcSQUID Neuromag Vectorview system. This system is comprised of 306 channels arranged in triplets of two orthogonally paired planar gradiometers and one magnetometer, which together measure magnetic field strength at 102 locations across the scalp. The MEG signal at each location was sampled at $601 \mathrm{~Hz}$ and bandpass filtered $(0.1-200 \mathrm{~Hz})$ during acquisition. Eye movements were recorded using electro-oculogram (EOG) electrodes, and four head position indicator (HPI) coils were attached to each subject's head to determine head position relative to the MEG sensors. To construct a head coordinate system, three fiduciary landmarks (nasion and bilateral preauricular points) were digitized using a three-dimensional digitizer (Polhemus), along with the locations of the HPI coils and a set of additional points on each subject's head. To aid in source localization, this coordinate system was aligned with an anatomic magnetic resonance image acquired from each subject (1.5 tesla Siemens Avanto Scanner) generated from of an average of two high-resolution T1weighted anatomical scans [MPRAGE sequence (TR, $2.7 \mathrm{~ms}$; TE, $3.3 \mathrm{~ms}$; flip angle, $7^{\circ}$ )].

$M E G$ postacquisition processing. Preprocessing and analysis of data were accomplished using MNE software developed at the Athinoula

A. Martinos Center for Biomedical Imaging (Charlestown, MA) (http:// www.nmr.mgh.harvard.edu/martinos/userInfo/data/sofMNE.php). Raw data were inspected visually and noisy channels were excluded from analysis. Two second epochs (plus a 200 ms prestimulus baseline) timelocked to stimulus onset were defined as the time window of interest. Waveforms within these time windows were additionally low-pass filtered at $30 \mathrm{~Hz}$, and the baseline period $200 \mathrm{~ms}$ before stimulus onset was subtracted from each epoch before averaging. Epochs containing EOG amplitudes exceeding $300 \mu \mathrm{V}$ or gradiometer amplitudes exceeding 2000 $\mathrm{fT} / \mathrm{cm}$ were excluded from the average. Averages for each event type (SH, $\mathrm{SM}, \mathrm{M}, \mathrm{FA}, \mathrm{CR}$ ) were calculated across trials within a run for an individual subject, and grand averages for each event type were calculated across runs for each subject. A minimum bin size of 25 total events per condition was set as a minimum threshold for a subject to be included in the analysis. To meet these minimum criteria, $\mathrm{SH}$ and $\mathrm{SM}$ responses were combined as a single event representing the correct identification of "old" items and averages were generated for this combined response category. Of the 15 controls and 16 patients who completed the MEG scan, one patient was excluded because of excessive noise artifact and one control and one patient were excluded because of inadequate bin sizes even after combining the SH and SM events as described above. This resulted in the inclusion of 14 controls and 14 patients in all subsequently described analyses.

MEG source space analysis. Three-dimensional cortical surface models were reconstructed from subjects' MR images using FreeSurfer software. Alignment of the MEG and MRI coordinate frames was accomplished manually by identifying the fiducial landmarks digitized on the subject's head using the high-resolution anatomic MR images described above. An ICP (iterative closest point) algorithm was then applied to fine-tune the locations of the additional digitized points. A single compartment (inner skull) BEM (boundary-element model) decimated to $\sim 5000$ dipole locations per hemisphere was used to calculate the forward solution. Inverse solutions of the MEG were calculated according to a MNE (minimum norm estimate) without depth weighting. All subjects' individual anatomical surfaces were averaged, and using a spherical morphing procedure, each subjects' inverse solution was projected onto this
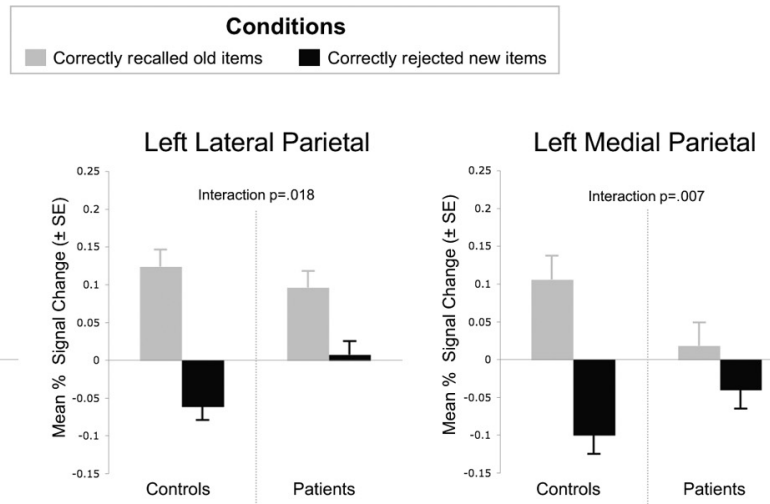

Right Lateral Parietal

Right Medial Parietal



Figure 2. Bar charts illustrating the event-related percentage BOLD signal change within the three bilateral regions of interest each region. Note the strong old-new modulation seen in control subjects across each examined region, a pattern that is diminia cohort.

common surface. This allowed within-group $t$ tests to be performed (Moon et al., 2007), to investigate differences in electromagnetic response to the old and new conditions at each vertex on the surface. These $t$ values were then visualized as within-group statistical maps of the oldnew difference, using a vertex-wise $\alpha$ of 0.05 .

To further evaluate the time course of old-new signal differences within the frontoparietal a priori ROIs, anatomically based ROIs were defined using an automated surface parcellation approach applied to the high-resolution magnetic resonance images (Fischl et al., 2004). Current estimates within these ROIs were then averaged to create group-level time courses for both old and new conditions. The so-called old-new effect consists of a greater electrical response to old items, relative to new items, beginning as early as $500 \mathrm{~ms}$ after stimulus presentation (Rugg and Curran, 2007). There are at least two temporally and regionally distinct aspects to this effect: one focused in the posterior parietal cortex (predominantly on the left), which extends from $\sim 500-800 \mathrm{~ms}$ after stimulus (also known as a P600 or late positive complex), and one focused in the right prefrontal cortex, extending from $\sim 600$ to $1600 \mathrm{~ms}$ after stimulus (also known as a late frontal effect) (Rugg and Nagy, 1989; Friedman, 1990; Tendolkar et al., 2000; Rugg and Curran, 2007). Hence signal differences between correctly recalled old items and newly presented items were examined in this context, with a focus on the 500-800 ms period in the left posterior parietal cortex, and the $600-1600 \mathrm{~ms}$ period in the right prefrontal cortex.

To determine whether old-new differences existed outside the frontoparietal ROIs, an exploratory, cortex-wide analysis was conducted across all vertices. To control for multiple comparisons, an uncorrected threshold of $p<0.001$ was used to identify significant clusters.

\section{Results}

\section{Behavioral data}

Behavioral data are summarized in Table 1. As reported previously (Weiss et al., 2008), during fMRI scans, control subjects were significantly more accurate in old-new recognition than patients with schizophrenia. When specifically examining the source accuracy of items deemed to be old (i.e., the percentage of 



Figure 3. Scatter plots showing the within-region relationship between activity to correctly identified old and new events $(\boldsymbol{a}-\boldsymbol{d})$ and the between-region relationship for the degree of old - new activity difference. In control subjects $(\boldsymbol{a}, \boldsymbol{c})$, note the near-unanimous location of the points below the diagonal, indicating greater activity to source hits than correct rejections. Note also the extremely high correlation between the overall degree of signal change for each event across the control cohort-whatever the level of activity to source hits, there is a consistent "activity buffer" that physiologically distinguishes these events from correctly rejected new items. This degree of old-new difference is essentially a constant in both the right frontal and right parietal cortices, with little evidence for correlation across the two regions at the individual subject level $(\boldsymbol{e})$. The pattern seen in patients with schizophrenia is entirely different $(\boldsymbol{b}, \boldsymbol{d})$. The tightly regulated activity relationship between source hits and correct rejections is abolished. We also see a large degree of variation around the diagonal, indicating that some patients show an old-new effect and some do not. Interestingly, the degree of old-new activity difference is strongly correlated across the two regions, with strong frontal lobe "activators" also being strong parietal lobe activators ( $f$ ). Finally, the degree of old-new activity is significantly correlated with performance, as noted by the performance-based shading of the points. Patients who are strong activators tend to show better recognition memory performance (dark shading) when compared with those who are weak activators (light shading).

correct source responses for old items labeled either "male" or "female"), there were no significant differences between the two groups. Analogous results were obtained when subjects underwent the task during MEG scanning.

\section{fMRI analyses}

Old versus new

Within the control subjects, comparison of the BOLD signal change associated with the correct identification of an item as old with that associated with the correct rejection of an item as new revealed a bilateral pattern of significant differences within the superior and middle dorsolateral prefrontal cortex (DLPFC), the lateral posterior parietal cortex (mostly within the angular gyrus and intraparietal sulcus), and the medial parietal cortex/precuneus (Fig. 1; supplemental Table 1, available at www.jneurosci. org as supplemental material). Subjects with schizophrenia showed a similar pattern of old versus new activation differences within the left hemisphere, with significant clusters located in the left DLPFC, posterior parietal cortex (angular gyrus and intraparietal sulcus), and the medial parietal cortex/precuneus. Unlike the control subjects, however, there were little to no old-new activation differences seen in the right hemisphere; indeed, the only clusters that met predetermined significance thresholds were deactivations located within the right superior, middle, and inferior temporal gyri. When the old-new effects seen in these two groups were compared directly, both the medial and lateral parietal cortices showed greater activity in control subjects, particularly within the right intraparietal sulcus and angular gyrus.

\section{Old versus miss}

The pattern of BOLD signal change differences between items correctly identified as old and items that were subsequently forgotten (i.e., misses) was similar to that seen in the old-new contrasts described above (supplemental Fig. 1, Table 2, available at www.jneurosci.org as supplemental material). 


\section{Current Estimates in Controls Left Parietal Lobe: Old vs New}

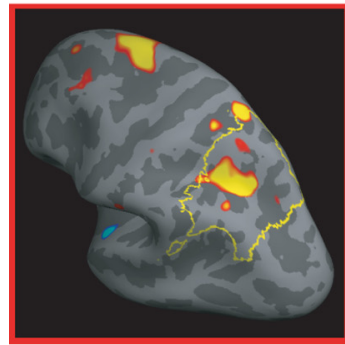

$440 \mathrm{~ms}$



$590 \mathrm{~ms}$

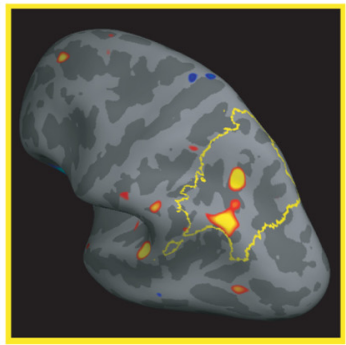

$720 \mathrm{~ms}$

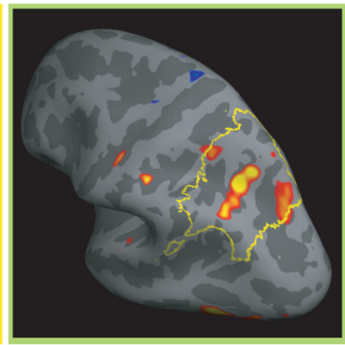

$770 \mathrm{~ms}$



$800 \mathrm{~ms}$
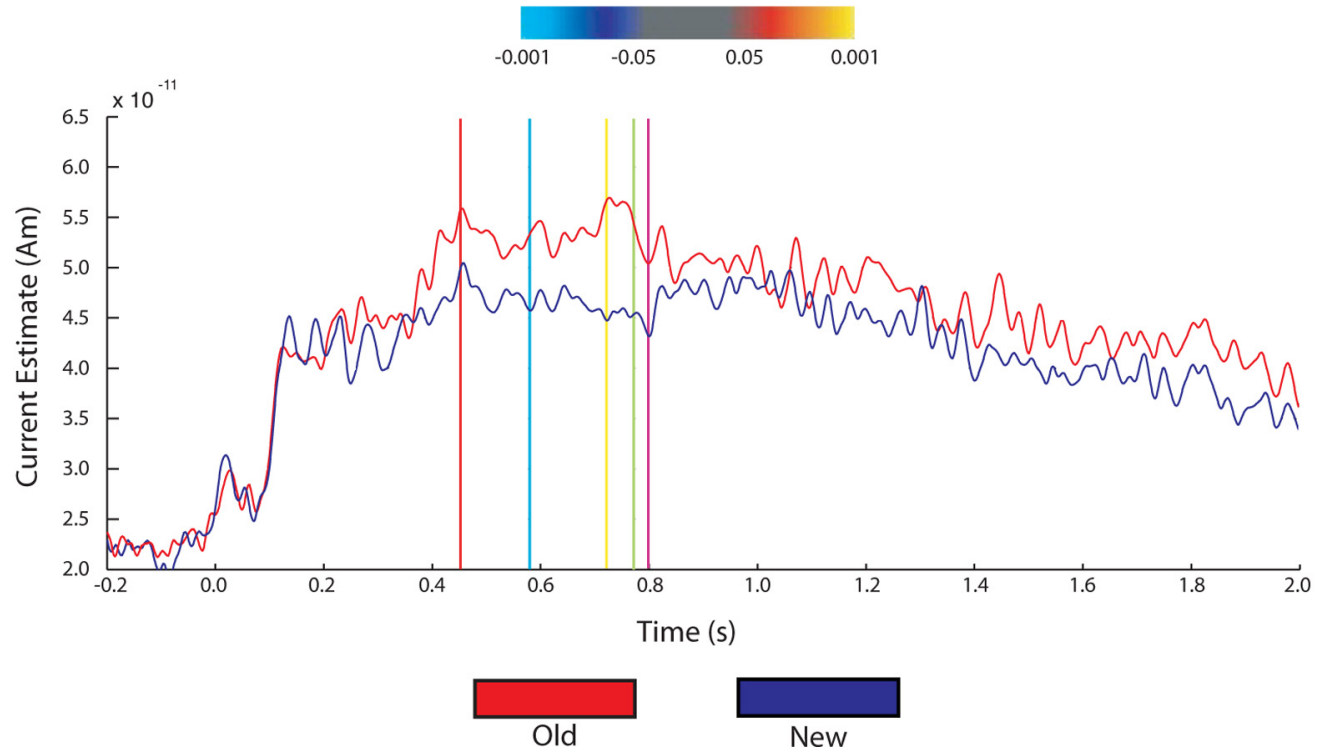

Figure 4. Source space analysis of left parietal old-new effect in controls. T-maps of the between condition difference in estimated current are displayed on inflated standard surfaces. Color coding represents statistical significance ( $p$ value) of this difference. The left posterior parietal cortex as anatomically defined by an automated parcellation approach is outlined in yellow. The group average signal (in ampere - meters) within this region is displayed below for both old and new conditions. Note the separation of the old and new time courses.

Source hits versus source misses

Neither control subjects nor patients with schizophrenia exhibited significant clusters of activation difference when correct versus incorrect source memory performance were compared directly. There were no significant regions demonstrating betweengroup activation differences for this contrast.

\section{Region of interest-based analyses}

The bilateral set of regions showing the greatest old-new effect in the healthy control population (DLPFC, lateral parietal cortex, and medial parietal cortex/precuneus) were identified as functionally defined regions of interest and the percentage BOLD signal change during the old and new conditions [i.e., ( $\mathrm{SH}$ plus $\mathrm{SM}$ ) vs CR] within each region was computed (Fig. 2). These values were then entered into a repeated measures ANOVA to look for main effects of condition [(SH plus SM) vs CR], hemisphere (left vs right), region (DLPFC, lateral parietal, and medial parietal/precuneus), and group (control vs schizophrenia) and the interactions between these effects. This analysis demonstrated a significant main effect for condition $\left(F_{(1,32)}=60.4 ; p<\right.$ $0.000001)$ and region $\left(F_{(2,64)}=8.86 ; p=0.0004\right)$, but not for diagnostic group $\left(F_{(1,32)}=0.18 ; p=0.68\right)$. Of relevance here, there was a significant group by condition interaction $\left(F_{(1,32)}=\right.$ $15.9 ; p=0.0004)$, as well as a group by condition by hemisphere by region interaction $\left(F_{(2,64)}=3.38 ; p=0.04\right)$. Follow-up analyses conducted within each of the six regions of interest indicated significant group by condition interactions in all regions except for the left DLPFC: left DLPFC $\left(F_{(1,32)}=2.67 ; p=0.112\right)$, right $\operatorname{DLPFC}\left(F_{(1,32)}=9.97 ; p=0.003\right)$, left lateral parietal $\left(F_{(1,32)}=\right.$ $6.23 ; p=0.018)$, right lateral parietal $\left(F_{(1,32)}=25.1 ; p=\right.$ $0.00002)$, left medial parietal $\left(F_{(1,32)}=8.40 ; p=0.007\right)$, and right medial parietal $\left(F_{(1,32)}=12.5 ; p=0.001\right)$. In each of the five significant regions, old-new differences were greater in controls than in patients.

\section{Frontal-parietal correlations}

To better understand the interrelationship between frontal and parietal old-new activation seen in these two groups, the ROIbased percentage signal change values were entered into a series of post hoc bivariate correlation analyses. In control subjects, there was a strong positive correlation between the degree of activation during correctly identified old items (source hits) and correctly rejected new items within a particular region (Fig. $3 a-c$ ). Thus, within both the right DLPFC $(r=0.92 ; p<0.000001)$ and right lateral parietal cortex $(r=0.92 ; p<0.000001)$, the degree of BOLD signal change during source hits was tightly correlated with the degree of BOLD signal change during correct rejections. However, the degree of activity between these two regions did not show a significant correlation in control subjects; neither for source hits $(r=0.46 ; p=0.06)$ nor for correct rejections $(r=$ $0.38 ; p=0.13$ ) (figures not shown). Overall, therefore, control subjects show a pattern in which there is consistent difference 


\section{Current Estimates in Patients Left Parietal Lobe: Old vs New}



$440 \mathrm{~ms}$



$590 \mathrm{~ms}$

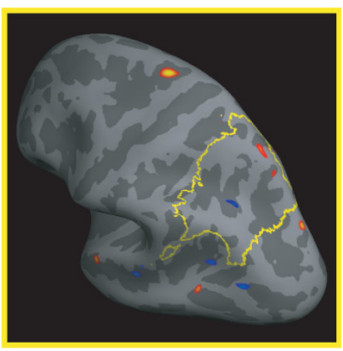

$720 \mathrm{~ms}$

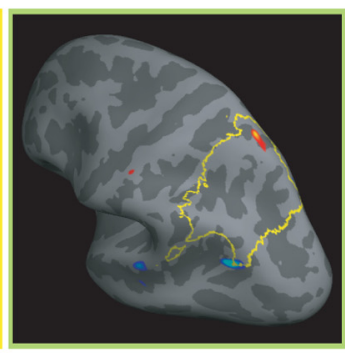

$770 \mathrm{~ms}$

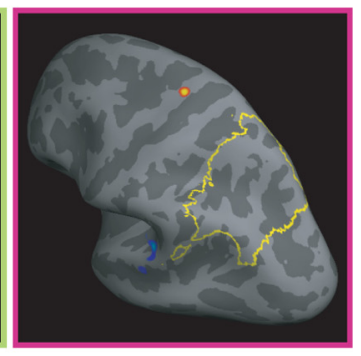

$800 \mathrm{~ms}$
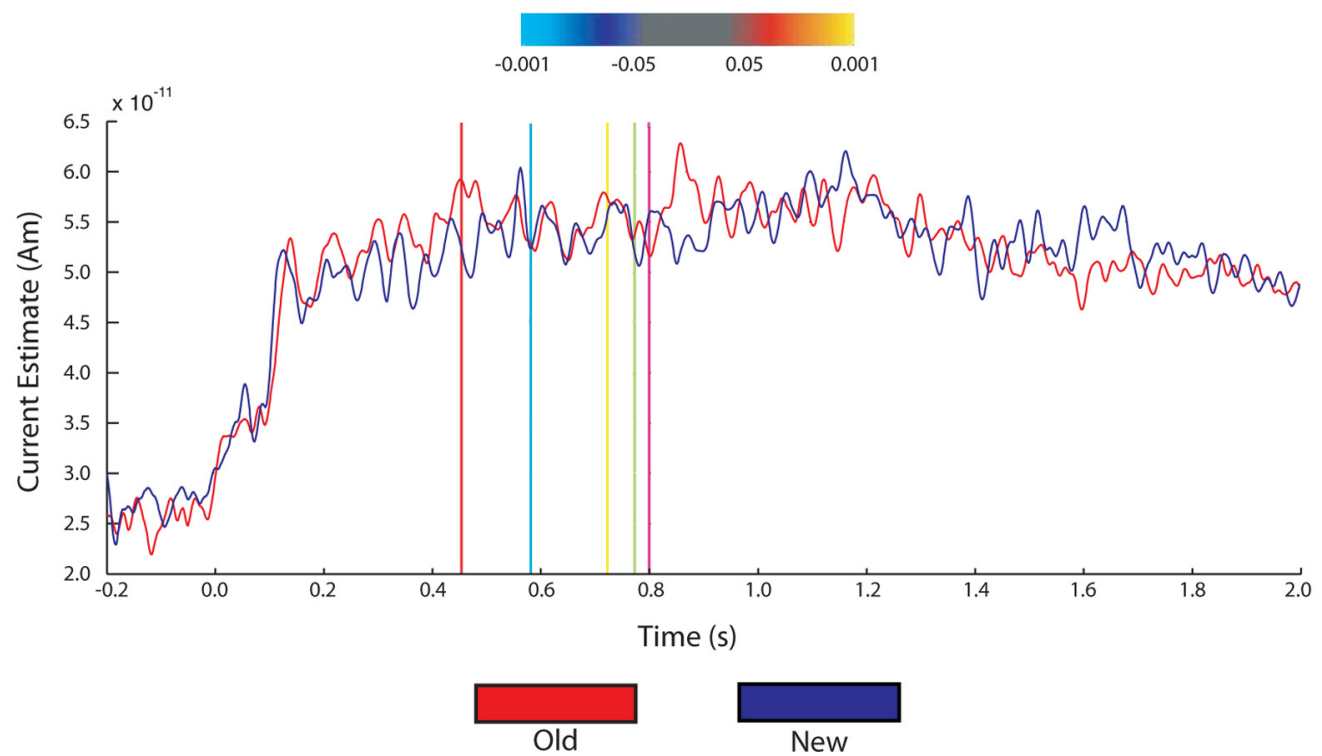

Figure 5. Source space analysis of left parietal old-new effect in patients. Group average signal within the left posterior parietal cortex is displayed on inflated cortical surfaces for both old and new conditions, as in Figure 4. Note the lack of separation of the old and new time courses.

between correctly identified old and new items in both frontal and parietal regions, but the degree of overall activity across these two regions is less tightly correlated.

The pattern seen in the patients with schizophrenia was precisely opposite to that seen in controls. In patients, there was no significant within-region correlation between source hits and correct rejections, neither in the frontal $(r=-0.03 ; p=0.92)$ nor lateral parietal $(r=0.30 ; p=0.24)$ cortices (Fig. $3 b, d)$. Thus, the degree of activation to one condition was not predictive of the degree of activity to the other condition within a single region. However, for a specific condition, there was a strong positive correlation between the frontal and parietal lobe activation pattern, true for both source hits $(r=0.67 ; p=0.003)$ and correct rejections ( $r=0.83 ; p=0.00005$ ) (figures not shown).

Finally, unlike control subjects in which the degree of old-new difference was practically a constant in both frontal and parietal regions (with no between-region correlation: $r=0.04 ; p=0.87$ ) (Fig. 3e), in patients there was an extraordinarily tight positive relationship between the degree of old-new differences seen in the right frontal lobe and that seen in the right parietal lobe at the individual subject level $(r=0.91 ; p=0.000001)$ (Fig. 3f). Similarly, correlations were found between the left parietal and right frontal region in patients $(r=0.65 ; p=0.004)$ but not controls $(r=0.00 ; p=1.00)$ (data not shown). Importantly, there was a strong relationship in patients between the degree of old-new activation difference and recognition memory performance (as measured by corrected recognition), in both the right frontal ( $r=$ $0.53 ; p=0.03)$ and right parietal $(r=0.50 ; p=0.04)$ regions of interest (figures not shown).

There was no correlation between the degree of old-new BOLD signal difference and clinical severity (as measured by both SAPS and SANS) in any of the bilaterally defined regions of interest (maximal correlation was between old-new difference in the left frontal region and SAPS score, $r=-0.27 ; p=0.29$ ). Similarly, no region showed a correlation between the degree of old-new BOLD signal difference and medication burden (based on chlorpromazine equivalents) (maximal correlation was in the left frontal region, $r=-0.43 ; p=0.09$ ).

\section{MEG source space analyses}

\section{Parietal lobe}

As anticipated, control subjects showed a strong old-new difference in estimated cortical electrical activity within the left posterior parietal lobe, centered on an area consistent with the intraparietal sulcus (Fig. 4). Separation in the electrical response began at $\sim 400 \mathrm{~ms}$ after stimulus and was sustained until $\sim 800 \mathrm{~ms}$ after stimulus. During this time window, tests of between-condition difference (old $>$ new) showed consistent evidence for statistically significant clusters (at $p<0.001$ ). Control subjects did not demonstrate the same degree of old-new separation within the right parietal cortex; occasional significant clusters were present, but they were small and widely interspersed in time. 


\section{Current Estimates in Controls Right Frontal Lobe: Old vs New}

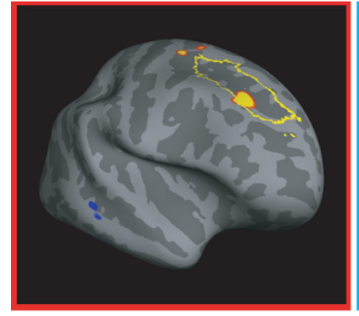

$960 \mathrm{~ms}$

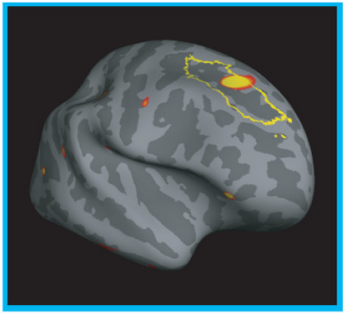

$1100 \mathrm{~ms}$

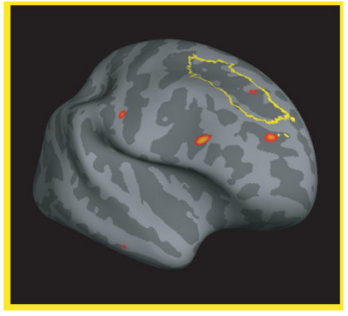

$1240 \mathrm{~ms}$

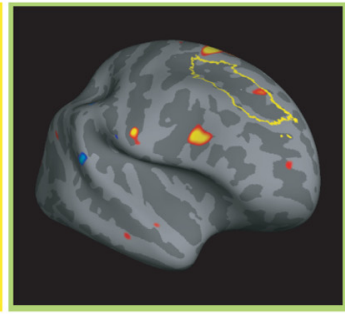

$1280 \mathrm{~ms}$

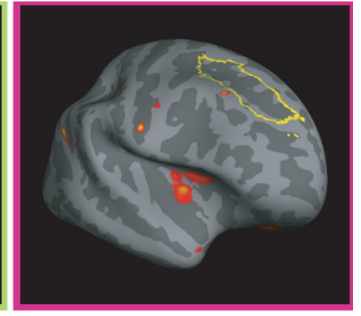

$1320 \mathrm{~ms}$
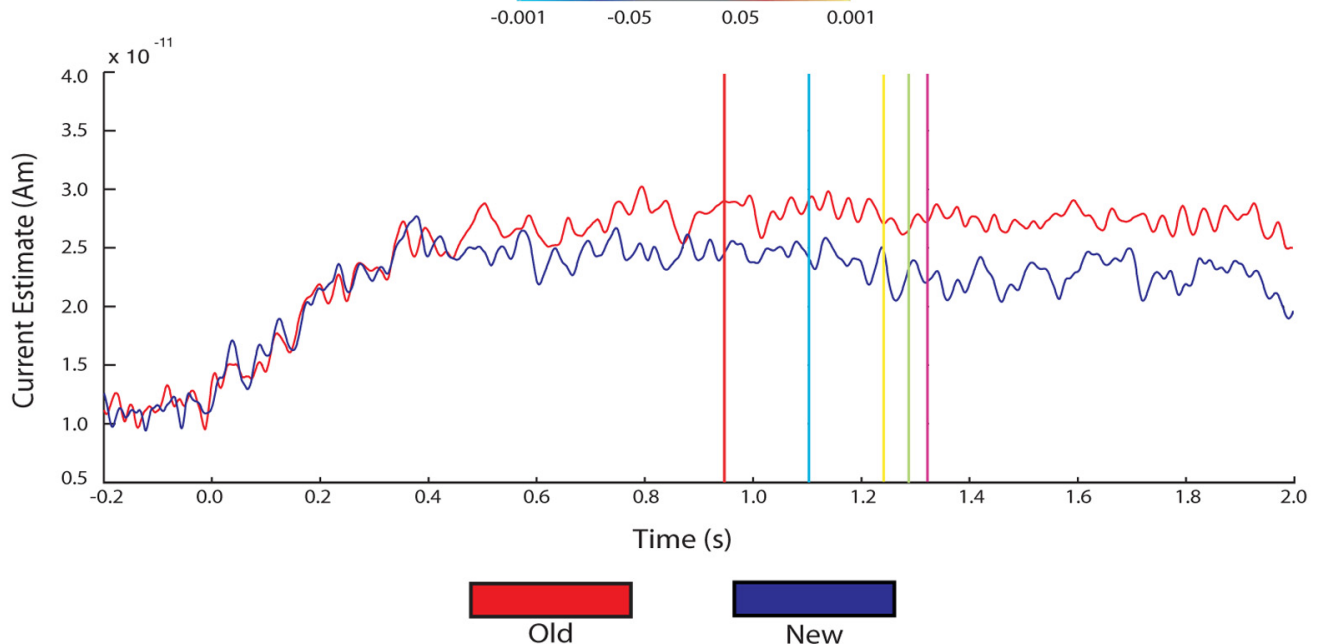

Figure 6. Source space analysis of right frontal old-new effect in controls. T-maps of the between condition difference in estimated current are displayed on inflated standard surfaces. Color coding represents statistical significance ( $p$ value) of this difference. The right superior frontal gyrus as anatomically defined by an automated parcellation approach is outlined in yellow. The group average signal within this region (in ampere • meters) is displayed below for both old and new conditions. Note, in controls, the strong old-new effect within the superior frontal gyrus that decreases in significance later over the time course.

Unlike the strong old-new separation seen in the controls, patients with schizophrenia showed no evidence for a parietal old-new effect, neither in the left nor right hemispheres. As seen in Figure 5, the estimated cortical electrical response to these two event types was mostly overlapping throughout the recording period. When compared with control subjects, patients demonstrated a similar degree of electrical activity to the old items, with an average current estimate of $\sim 5.4$ to $5.5 \times 10^{11}$ ampere $\cdot$ meters (Am) in both groups during the $500-800 \mathrm{~ms}$ poststimulus time window. But in contradistinction to control subjects, patients did not downregulate this activity during the correct rejection of new items; their average current estimates in response to these events remained at $\sim 5.5 \times 10^{11} \mathrm{Am}$, whereas it was nearly a full unit lower in control subjects.

\section{Frontal lobe}

Control subjects also demonstrated a significant old-new effect within the right superior frontal lobe (Fig. 6). The electrical response to these two events diverged at $\sim 600 \mathrm{~ms}$ after stimulus and showed a sustained difference throughout the remainder of the time course. Statistically significant clusters were evident at several points throughout the period, most substantially during the $900-1200 \mathrm{~ms}$ poststimulus time frame.

Patients with schizophrenia did not demonstrate this right frontal old-new effect (Fig. 7). In fact, beginning at $\sim 700 \mathrm{~ms}$ after stimulus, there was a reversal pattern seen in this cohort, with correctly rejected new items eliciting a more robust electrical response than correctly recognized old items. This new-old dif- ference was apparent throughout the remainder of the time course, with statistically significant clusters becoming most apparent during the $1200-1300 \mathrm{~ms}$ poststimulus time frame. Once again, the response to old items was relatively similar when compared between the two groups, whereas the response to new items showed a more marked amplitude difference.

\section{Superior and middle temporal gyri}

Although the patients with schizophrenia did not demonstrate the expected old-new differences within the frontoparietal regions of a priori interest, an exploratory inspection of cortical regions outside of these areas revealed a dramatic pattern of activity difference within the auditory-related cortical regions bilaterally (Fig. 8). Within the left superior and middle temporal lobes, patients showed a robust pattern of new $>$ old activity, which began at $\sim 400 \mathrm{~ms}$ after stimulus and terminated at $\sim 900$ ms after stimulus. At about that point, the patients then show a robust old $>$ new effect within the corresponding regions in the right hemisphere, an effect that was sustained until $\sim 1300 \mathrm{~ms}$ after stimulus. Control subjects did not demonstrate these patterns, with statistically equivalent old and new responses within the temporal cortices of both hemispheres (data not shown).

\section{Discussion}

Differentiating new from previously presented items is a basic and critically important element of recognition memory, one that appears to be disrupted in schizophrenia. Using multimodal imaging techniques, we have demonstrated in this investigation 


\section{Current Estimates in Patients Right Frontal Lobe: Old vs New}

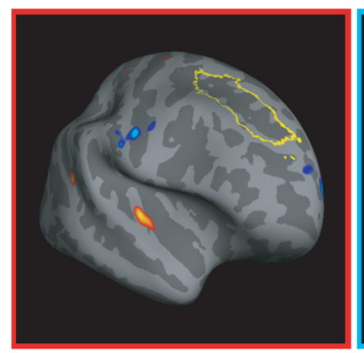

$960 \mathrm{~ms}$

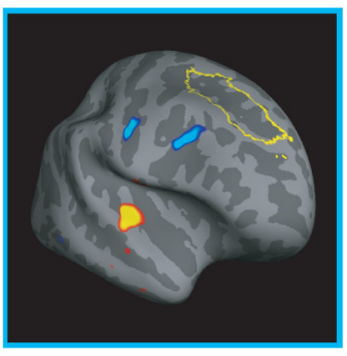

$1100 \mathrm{~ms}$

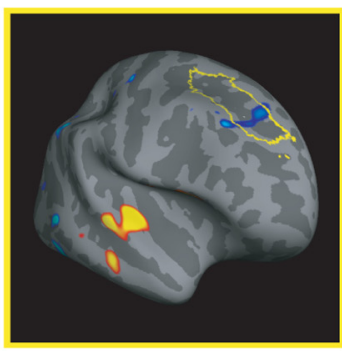

$1240 \mathrm{~ms}$

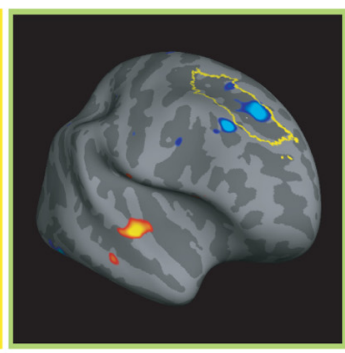

$1280 \mathrm{~ms}$

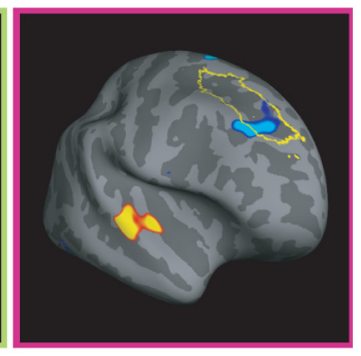

$1320 \mathrm{~ms}$


Figure 7. Source space analysis of right frontal old-new effect in patients. Group average signal within the right superior frontal gyrus is displayed on inflated cortical surfaces for both old and new conditions, as in Figure 6. Note that, in patients, there is a complete reversal of the effect seen in controls over the same time course. Rather, patients exhibit a "new- old" effect that increases in significance across the time course.

that the neural physiology underlying old-new item distinction differs substantially between schizophrenia patients and healthy control subjects. The two principal findings are summarized as follows: (1) In the context of poorer recognition memory performance, patients with schizophrenia showed either a diminished or absent old-new effect within the frontoparietal network, particularly within the right hemisphere. Those patients who did show a "normal" physiological response pattern (i.e., greater BOLD signal for old vs new) also showed a normal level of recognition memory performance. (2) As seen with the finer temporal resolution of MEG, control subjects exhibited a sequential pattern of old $>$ new electrical activity first in the left posterior parietal cortex and then right prefrontal cortex, consistent with previous reports, whereas no such differences were found in patients.

The strong frontoparietal old-new effect in control subjects is consistent with a relatively large fMRI literature documenting the importance of these regions in normal episodic memory processes (Nolde et al., 1998; Konishi et al., 2000; Dobbins et al., 2003; Leube et al., 2003; Maril et al., 2003; Kahn et al., 2004; Wheeler and Buckner, 2004; Hofer et al., 2007) (for relevant recent reviews, see Wagner et al., 2005; Skinner and Fernandes, 2007; Cabeza et al., 2008). Previous fMRI studies of the old-new effect in patients with schizophrenia have also identified aberrant modulation of both frontal and parietal lobe regions, although both frontoparietal hypoactivity (Weiss et al., 2006) and hyperactivity (Ragland et al., 2004) have been associated with task performance.

Consistent with a large body of previous ERP research (Rugg and Curran, 2007), as well as two previous MEG reports (Tendolkar et al., 2000; Walla et al., 2005), healthy control subjects showed a clear differential response to old and new items beginning first in the inferior parietal cortex at $\sim 400-500 \mathrm{~ms}$ after stimulus, followed by the right superior frontal gyrus (400-1600 ms after stimulus). In contrast to the healthy subjects in these studies, as well as our control population, the patients in our study showed neither the left posterior parietal nor the right superior prefrontal old-new effects. Although to our knowledge this represents the first study to use MEG to examine this effect in patients with schizophrenia, a body of ERP literature on this topic does exist (Kayser et al., 1999; Matsuoka et al., 1999; Guillem et al., 2001, 2003; Matsumoto et al., 2001; Tendolkar et al., 2002; Kim et al., 2004). Of these, the study by Tendolkar et al. is most similar to the present report in that it tested recognition memory for words using discrete study and test blocks. In comparison with a control population, they found that patients with schizophrenia demonstrated temporally truncated old-new effects (frontal and parietal) in association with consciously recalled responses, and a lack of a parietal lobe old-new effect to items that were only associated with a sense of familiarity. 


\section{Current Estimates in Patients Temporal Lobes: Old vs New}

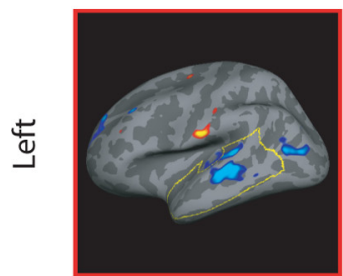

$570 \mathrm{~ms}$

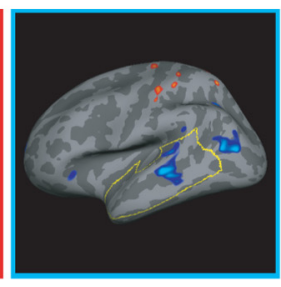

$680 \mathrm{~ms}$

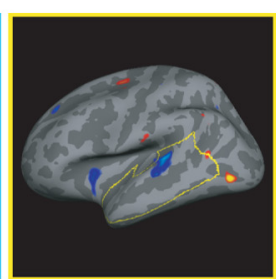

$820 \mathrm{~ms}$

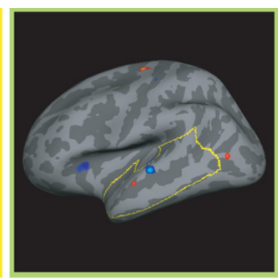

$920 \mathrm{~ms}$

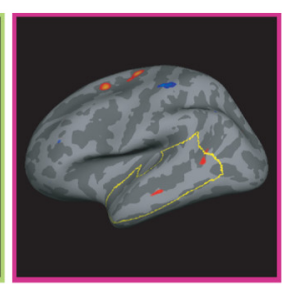

$1090 \mathrm{~ms}$

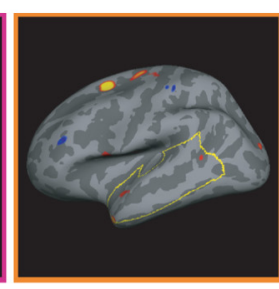

$1200 \mathrm{~ms}$
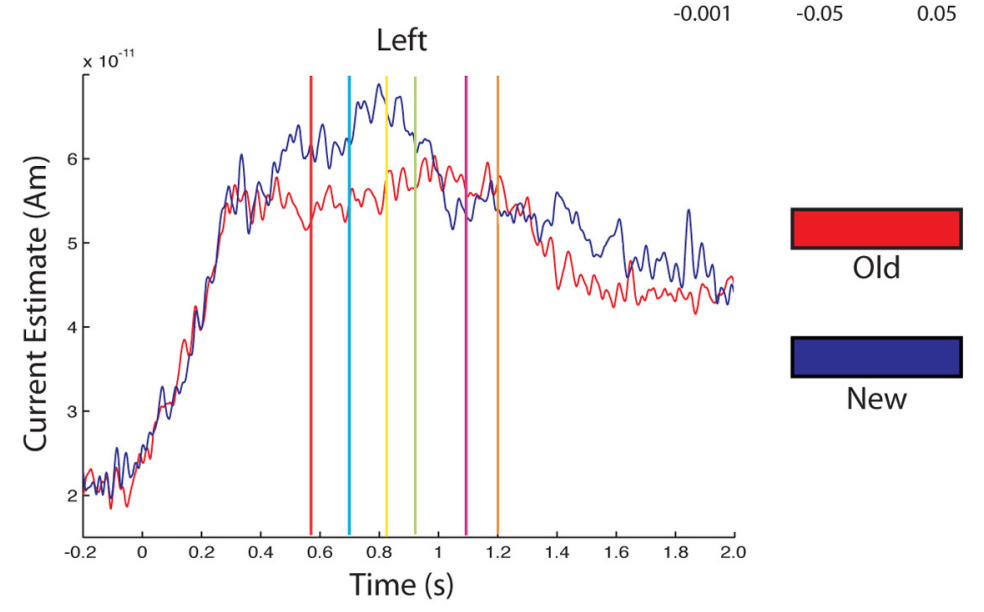

0.001

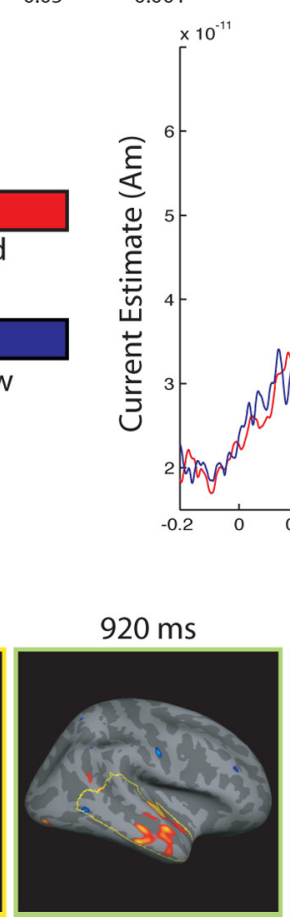

$820 \mathrm{~ms}$
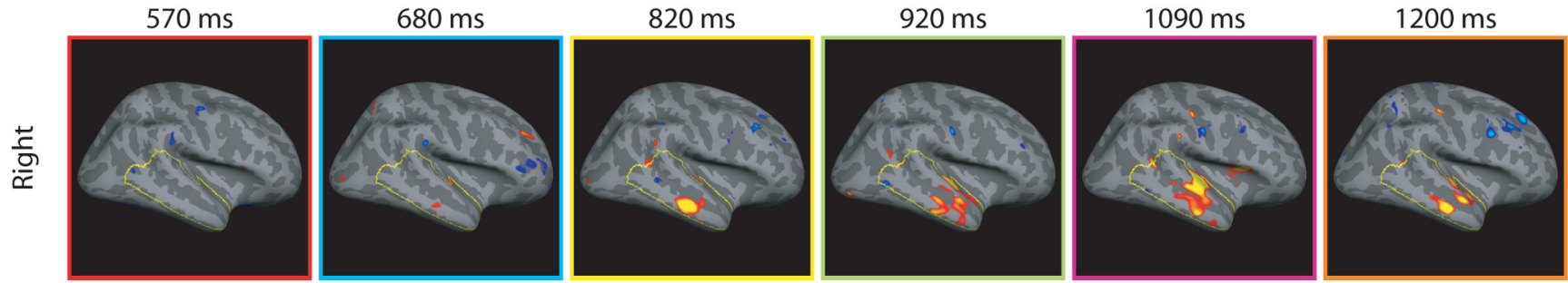

Figure 8. Source space analysis of left and right temporal lobe (patients). T-maps of the between-condition difference in estimated current are displayed on inflated standard surfaces. Color coding represents statistical significance ( $p$ value) of this difference. The left and right superior and middle temporal gyri as anatomically defined by an automated parcellation approach are outlined in yellow. The group average signals within both left and right regions (in ampere $\cdot$ meters) are displayed below for both old and new conditions. Note the reverse patterns of activity occurring between the two hemispheres in which the left displays a new-old effect occurring earlier than the old-new effect in the right hemisphere of the patients.

Collectively, the present fMRI and MEG findings are consistent with markedly different distributions of regional specialization necessary to complete the task in patients versus controls. In healthy subjects, the old $>$ new electrical signal that we and others have observed in the left posterior parietal cortex has generally been thought to reflect recollective processes, as the effect has been shown to be sensitive to accurate source judgment as well as linked to the subjective experience of conscious retrieval (compared with familiarity) using the remember-know paradigm (Düzel et al., 1997; Donaldson and Rugg, 1999; Curran, 2000; Curran et al., 2001; Duarte et al., 2004). The predominantly rightsided late frontal old-new effect has also been generally linked to recollective processes, such as those required for accurate source or associative memory (Wilding and Rugg, 1996; Allan and Rugg, 1998; Allan et al., 2000). A number of studies have alternatively or additionally linked this physiologic marker to postretrieval monitoring, with some recent evidence suggesting that the effect is more associated to general monitoring or decision making, rather than being a retrieval-specific component (Dobbins and Han, 2006; Hayama et al., 2008). Such goal-directed activity at different times, in different regions, even in different hemi- spheres, suggests a highly regionally specialized use of information. Similarly, the bilateral activation of both frontal and parietal regions coupled with a lack of correlation between them, as seen with $\mathrm{fMRI}$, suggests that these regions are each engaged in components of the old-new item distinction process, but that those components differ between regions.

In patients, however, the lack of distinction between old and new items as seen in the left parietal and right frontal MEG recordings during their respective critical time periods suggests a failure of these regions to distinguish old and new items (despite their engagement in the task, as suggested by the rise in signal above baseline). As suggested by their highly correlated BOLD signal, however, these regions may be working in a coordinated way to facilitate task performance, which is, after all, intact in the events included in this analysis. Furthermore, the temporal lobe MEG findings, unique to patients, suggests that recruitment of another region (one not normally associated with old-new item distinction) may be necessary for successful performance in patients. This explanation would be consistent with the work of Ragland and colleagues (Ragland et al., 2004; Ragland et al., 2006; Wolf et al., 2007), who have suggested that the enhanced fronto- 
temporal connectivity and temporal lobe hyperactivity seen during recognition memory in patients with schizophrenia, are signs of a (ineffective) compensatory mechanism. Although speculative, it may also be possible that the absence of the frontoparietal old-new effect diminishes the sense of confidence that patients have in their response, a widely reported finding within the schizophrenia memory literature (Bacon et al., 2001; Moritz and Woodward, 2002, 2006; Moritz et al., 2003, 2005; Kircher et al., 2007). Indeed, individuals with parietal lobe lesions have been found to have intact episodic memory (including source memory) but impaired confidence in or vividness of the recollected material (Berryhill et al., 2007; Davidson et al., 2008; Simons et al., 2008). Additional study, directly measuring memory confidence in conjunction with neuroimaging data in patients with schizophrenia, would be necessary to test this hypothesis.

It is important to note that frontoparietal activation may not be specific to episodic memory performance. These regions are in fact associated with a wide variety of cognitive tasks, including working memory, executive function, and conscious visual perception (Naghavi and Nyberg, 2005). It remains possible that the frontoparietal abnormalities seen in the present study are related to a broader and cross-cutting cognitive construct, such as cognitive control (MacDonald et al., 2000) or the allocation of attention (Cabeza et al., 2008), rather than specifically and solely to episodic memory performance. Indeed, this may help explain why deficient activation of these areas has been associated with a number of cognitive impairments in schizophrenia, including transitive inference (Ongür et al., 2006), semantic recall (Assaf et al., 2006), working memory (Barch and Csernansky, 2007), visual attention (Gur et al., 2007), and response suppression (Ford et al., 2004).

The inclusion of medicated patients with schizophrenia represents the primary limitation of the present study. As dopamine may play a role in the detection and response to novelty (Lisman and Grace, 2005), we cannot fully exclude the possibility that medication with dopamine antagonists may have contributed to the abnormalities in stimulus-related signal reported here. This seems unlikely for a number of reasons. First, there was no correlation between medication burden and either memory performance or the degree of old-new BOLD signal difference in any of the six ROIs examined. Second, based on a recent review of the literature it appears that the longitudinal use of antipsychotic medications is more often associated with normalization of cerebral activity, although substantial methodological differences preclude confident conclusions at this time (Davis et al., 2005). Furthermore, the pattern of task-related dorsolateral prefrontal cortex hypoactivity seen in patients with schizophrenia has been shown to be unaffected by the initiation of antipsychotic medication (Snitz et al., 2005).

This issue notwithstanding, the results presented here indicate an important physiological correlate of aberrant cognitive performance in patients with schizophrenia. Additional work is necessary to better understand the interindividual variance in performance and physiology seen within the patient cohort. In particular, the potential role of genetic polymorphisms now known to affect the activity of this frontoparietal circuit (Tan et al., 2007a) would be of substantial interest.

\section{References}

Allan K, Rugg MD (1998) Neural correlates of cued recall with and without retrieval of source memory. Neuroreport 9:3463-3466.

Allan K, Robb WG, Rugg MD (2000) The effect of encoding manipulations on neural correlates of episodic retrieval. Neuropsychologia 38:1188-1205.
Andreasen NC (1983) The Scale for the Assessment of Negative Symptoms (SANS). Iowa City, IA: University of Iowa.

Andreasen NC (1984) The Scale for the Assessment of Positive Symptoms (SAPS). Iowa City, IA: University of Iowa.

Assaf M, Rivkin PR, Kuzu CH, Calhoun VD, Kraut MA, Groth KM, Yassa MA, Hart J Jr, Pearlson GD (2006) Abnormal object recall and anterior cingulate overactivation correlate with formal thought disorder in schizophrenia. Biol Psychiatry 59:452-459.

Bacon E, Danion JM, Kauffmann-Muller F, Bruant A (2001) Consciousness in schizophrenia: a metacognitive approach to semantic memory. Conscious Cogn 10:473-484.

Barbas H, Zikopoulos B (2007) The prefrontal cortex and flexible behavior. Neuroscientist 13:532-545.

Barch DM, Csernansky JG (2007) Abnormal parietal cortex activation during working memory in schizophrenia: verbal phonological coding disturbances versus domain-general executive dysfunction. Am J Psychiatry 164:1090-1098.

Berryhill ME, Phuong L, Picasso L, Cabeza R, Olson IR (2007) Parietal lobe and episodic memory: bilateral damage causes impaired free recall of autobiographical memory. J Neurosci 27:14415-14423.

Blair JR, Spreen O (1989) Predicting premorbid IQ: a revision of the National Adult Reading Test. Clin Neuropsychol 3:129-136.

Cabeza R, Ciaramelli E, Olson IR, Moscovitch M (2008) The parietal cortex and episodic memory: an attentional account. Nat Rev Neurosci 9:613-625.

Cannistraro PA, Wright CI, Wedig MM, Martis B, Shin LM, Wilhelm S, Rauch SL (2004) Amygdala responses to human faces in obsessivecompulsive disorder. Biol Psychiatry 56:916-920.

Cox RW (1996) AFNI: software for analysis and visualization of functional magnetic resonance neuroimages. Comput Biomed Res 29:162-173.

Curran T (2000) Brain potentials of recollection and familiarity. Mem Cogn 28:923-938.

Curran T, Schacter DL, Johnson MK, Spinks R (2001) Brain potentials reflect behavioral differences in true and false recognition. J Cogn Neurosci 13:201-216.

Dale AM, Fischl B, Sereno MI (1999) Cortical surface-based analysis. I. Segmentation and surface reconstruction. Neuroimage 9:179-194.

Davidson PS, Anaki D, Ciaramelli E, Cohn M, Kim AS, Murphy KJ, Troyer AK, Moscovitch M, Levine B (2008) Does lateral parietal cortex support episodic memory? Evidence from focal lesion patients. Neuropsychologia 46:1743-1755.

Davis CE, Jeste DV, Eyler LT (2005) Review of longitudinal functional neuroimaging studies of drug treatments in patients with schizophrenia. Schizophr Res 78:45-60.

Dobbins IG, Han S (2006) Isolating rule- versus evidence-based prefrontal activity during episodic and lexical discrimination: a functional magnetic resonance imaging investigation of detection theory distinctions. Cereb Cortex 16:1614-1622.

Dobbins IG, Rice HJ, Wagner AD, Schacter DL (2003) Memory orientation and success: separable neurocognitive components underlying episodic recognition. Neuropsychologia 41:318-333.

Donaldson DI, Rugg MD (1999) Event-related potential studies of associative recognition and recall: electrophysiological evidence for context dependent retrieval processes. Brain Res Cogn Brain Res 8:1-16.

Duarte A, Ranganath C, Winward L, Hayward D, Knight RT (2004) Dissociable neural correlates for familiarity and recollection during the encoding and retrieval of pictures. Brain Res Cogn Brain Res 18:255-272.

Düzel E, Yonelinas AP, Mangun GR, Heinze HJ, Tulving E (1997) Eventrelated brain potential correlates of two states of conscious awareness in memory. Proc Natl Acad Sci U S A 94:5973-5978.

First MB, Spitzer RL, Gibbon M, Williams JBW (1995) Stuctured Clinical Interview for DSM-IV Axis I Disorders, Patient Edition (SCID-P), version 2. New York: New York State Psychiatric Institute, Biometrics Research.

Fischl B, Sereno MI, Dale AM (1999a) Cortical surface-based analysis. II: Inflation, flattening, and a surface-based coordinate system. Neuroimage 9:195-207.

Fischl B, Sereno MI, Tootell RB, Dale AM (1999b) High-resolution intersubject averaging and a coordinate system for the cortical surface. Hum Brain Mapp 8:272-284.

Fischl B, van der Kouwe A, Destrieux C, Halgren E, Ségonne F, Salat DH, Busa E, Seidman LJ, Goldstein J, Kennedy D, Caviness V, Makris N, Rosen B, 
Dale AM (2004) Automatically parcellating the human cerebral cortex. Cereb Cortex 14:11-22.

Ford JM, Gray M, Whitfield SL, Turken AU, Glover G, Faustman WO, Mathalon DH (2004) Acquiring and inhibiting prepotent responses in schizophrenia: event-related brain potentials and functional magnetic resonance imaging. Arch Gen Psychiatry 61:119-129.

Friedman D (1990) Cognitive event-related potential components during continuous recognition memory for pictures. Psychophysiology $27: 136-148$.

Fuster JM (2006) The cognit: a network model of cortical representation. Int J Psychophysiol 60:125-132.

Green MF (2006) Cognitive impairment and functional outcome in schizophrenia and bipolar disorder. J Clin Psychiatry 67 [Suppl 9]:3-8; discussion $36-42$.

Guillem F, Bicu M, Hooper R, Bloom D, Wolf MA, Messier J, Desautels R, Debruille JB (2001) Memory impairment in schizophrenia: a study using event-related potentials in implicit and explicit tasks. Psychiatry Res 104:157-173.

Guillem F, Bicu M, Pampoulova T, Hooper R, Bloom D, Wolf MA, Messier J, Desautels R, Todorov C, Lalonde P, Debruille JB (2003) The cognitive and anatomo-functional basis of reality distortion in schizophrenia: a view from memory event-related potentials. Psychiatry Res 117:137-158.

Gur RE, Turetsky BI, Loughead J, Snyder W, Kohler C, Elliott M, Pratiwadi R, Ragland JD, Bilker WB, Siegel SJ, Kanes SJ, Arnold SE, Gur RC (2007) Visual attention circuitry in schizophrenia investigated with oddball event-related functional magnetic resonance imaging. Am J Psychiatry 164:442-449.

Hagler DJ Jr, Saygin AP, Sereno MI (2006) Smoothing and cluster thresholding for cortical surface-based group analysis of fMRI data. Neuroimage 33:1093-1103.

Hayama HR, Johnson JD, Rugg MD (2008) The relationship between the right frontal old/new ERP effect and post-retrieval monitoring: specific or non-specific? Neuropsychologia 46:1211-1223.

Heinrichs RW, Goldberg JO, Miles AA, McDermid Vaz S (2008) Predictors of medication competence in schizophrenia patients. Psychiatry Res 157:47-52.

Hofer A, Siedentopf CM, Ischebeck A, Rettenbacher MA, Widschwendter CG, Verius M, Golaszewski SM, Koppelstaetter F, Felber S, Wolfgang Fleischhacker W (2007) The neural regions sustaining episodic encoding and recognition of objects. Brain Cogn 63:159-166.

Jeste SD, Patterson TL, Palmer BW, Dolder CR, Goldman S, Jeste DV (2003) Cognitive predictors of medication adherence among middle-aged and older outpatients with schizophrenia. Schizophr Res 63:49-58.

Kahn I, Davachi L, Wagner AD (2004) Functional-neuroanatomic correlates of recollection: implications for models of recognition memory. J Neurosci 24:4172-4180.

Kayser J, Bruder GE, Friedman D, Tenke CE, Amador XF, Clark SC, Malaspina D, Gorman JM (1999) Brain event-related potentials (ERPs) in schizophrenia during a word recognition memory task. Int J Psychophysiol 34:249-265.

Keefe RS, Bilder RM, Davis SM, Harvey PD, Palmer BW, Gold JM, Meltzer HY, Green MF, Capuano G, Stroup TS, McEvoy JP, Swartz MS, Rosenheck RA, Perkins DO, Davis CE, Hsiao JK, Lieberman JA (2007) Neurocognitive effects of antipsychotic medications in patients with chronic schizophrenia in the CATIE Trial. Arch Gen Psychiatry 64:633-647.

Kim MS, Kwon JS, Kang SS, Youn T, Kang KW (2004) Impairment of recognition memory in schizophrenia: event-related potential study using a continuous recognition task. Psychiatry Clin Neurosci 58:465-472.

Kircher TT, Koch K, Stottmeister F, Durst V (2007) Metacognition and reflexivity in patients with schizophrenia. Psychopathology 40:254-260.

Konishi S, Wheeler ME, Donaldson DI, Buckner RL (2000) Neural correlates of episodic retrieval success. Neuroimage 12:276-286.

Leube DT, Erb M, Grodd W, Bartels M, Kircher TT (2003) Successful episodic memory retrieval of newly learned faces activates a left frontoparietal network. Brain Res Cogn Brain Res 18:97-101.

Lisman JE, Grace AA (2005) The hippocampal-VTA loop: controlling the entry of information into long-term memory. Neuron 46:703-713.

MacDonald AW 3rd, Cohen JD, Stenger VA, Carter CS (2000) Dissociating the role of the dorsolateral prefrontal and anterior cingulate cortex in cognitive control. Science 288:1835-1838.

Maril A, Simons JS, Mitchell JP, Schwartz BL, Schacter DL (2003) Feeling- of-knowing in episodic memory: an event-related fMRI study. Neuroimage 18:827-836.

Matsumoto K, Matsuoka H, Yamazaki H, Sakai H, Kato T, Miura N, Nakamura M, Osakabe K, Saito H, Ueno T, Sato M (2001) Impairment of an event-related potential correlate of memory in schizophrenia: effects of immediate and delayed word repetition. Clin Neurophysiol 112:662-673.

Matsuoka H, Matsumoto K, Yamazaki H, Sakai H, Miwa S, Yoshida S, Numachi Y, Saito H, Ueno T, Sato M (1999) Lack of repetition priming effect on visual event-related potentials in schizophrenia. Biol Psychiatry 46:137-140.

Moon SY, Barton JJ, Mikulski S, Polli FE, Cain MS, Vangel M, Hämäläinen MS, Manoach DS (2007) Where left becomes right: a magnetoencephalographic study of sensorimotor transformation for antisaccades. Neuroimage 36:1313-1323.

Moritz S, Woodward TS (2002) Memory confidence and false memories in schizophrenia. J Nerv Ment Dis 190:641-643.

Moritz S, Woodward TS (2006) The contribution of metamemory deficits to schizophrenia. J Abnorm Psychol 115:15-25.

Moritz S, Woodward TS, Ruff CC (2003) Source monitoring and memory confidence in schizophrenia. Psychol Med 33:131-139.

Moritz S, Woodward TS, Whitman JC, Cuttler C (2005) Confidence in errors as a possible basis for delusions in schizophrenia. J Nerv Ment Dis 193:9-16.

Naghavi HR, Nyberg L (2005) Common fronto-parietal activity in attention, memory, and consciousness: shared demands on integration? Conscious Cogn 14:390-425.

Nolde SF, Johnson MK, D’Esposito M (1998) Left prefrontal activation during episodic remembering: an event-related fMRI study. Neuroreport 9:3509-3514.

Ongür D, Cullen TJ, Wolf DH, Rohan M, Barreira P, Zalesak M, Heckers S (2006) The neural basis of relational memory deficits in schizophrenia. Arch Gen Psychiatry 63:356-365.

Ragland JD, Gur RC, Valdez J, Turetsky BI, Elliott M, Kohler C, Siegel S, Kanes S, Gur RE (2004) Event-related fMRI of frontotemporal activity during word encoding and recognition in schizophrenia. Am J Psychiatry 161:1004-1015.

Ragland JD, Valdez JN, Loughead J, Gur RC, Gur RE (2006) Functional magnetic resonance imaging of internal source monitoring in schizophrenia: recognition with and without recollection. Schizophr Res 87:160-171.

Ragland JD, Yoon J, Minzenberg MJ, Carter CS (2007) Neuroimaging of cognitive disability in schizophrenia: search for a pathophysiological mechanism. Int Rev Psychiatry 19:417-427.

Ranganath C, Minzenberg MJ, Ragland JD (2008) The cognitive neuroscience of memory function and dysfunction in schizophrenia. Biol Psychiatry $64: 18-25$.

Roffman JL, Goff DC, Schacter DL, Ellis CB, Stufflebeam S, Hamalainen MS, Duff M, Weiss AP (2009) Altered prefrontal-parietal physiology during old-new item recognition in schizophrenia: a multimodal neuroimaging investigation. Schizophr Bull 35:177.

Rugg MD, Curran T (2007) Event-related potentials and recognition memory. Trends Cogn Sci 11:251-257.

Rugg MD, Nagy ME (1989) Event-related potentials and recognition memory for words. Electroencephalogr Clin Neurophysiol 72:395-406.

Simon AE, Cattapan-Ludewig K, Zmilacher S, Arbach D, Gruber K, Dvorsky DN, Roth B, Isler E, Zimmer A, Umbricht D (2007) Cognitive functioning in the schizophrenia prodrome. Schizophr Bull 33:761-771.

Simons JS, Peers PV, Hwang DY, Ally BA, Fletcher PC, Budson AE (2008) Is the parietal lobe necessary for recollection in humans? Neuropsychologia 46:1185-1191.

Skinner EI, Fernandes MA (2007) Neural correlates of recollection and familiarity: a review of neuroimaging and patient data. Neuropsychologia 45:2163-2179.

Snitz BE, MacDonald A 3rd, Cohen JD, Cho RY, Becker T, Carter CS (2005) Lateral and medial hypofrontality in first-episode schizophrenia: functional activity in a medication-naive state and effects of short-term atypical antipsychotic treatment. Am J Psychiatry 162:2322-2329.

Tan HY, Chen Q, Sust S, Buckholtz JW, Meyers JD, Egan MF, Mattay VS, Meyer-Lindenberg A, Weinberger DR, Callicott JH (2007a) Epistasis between catechol-O-methyltransferase and type II metabotropic glutamate receptor 3 genes on working memory brain function. Proc Natl Acad Sci U S A 104:12536-12541. 
Tan HY, Callicott JH, Weinberger DR (2007b) Dysfunctional and compensatory prefrontal cortical systems, genes and the pathogenesis of schizophrenia. Cereb Cortex 17 [Suppl 1]:i171-i181.

Tendolkar I, Rugg M, Fell J, Vogt H, Scholz M, Hinrichs H, Heinze HJ (2000) A magnetoencephalographic study of brain activity related to recognition memory in healthy young human subjects. Neurosci Lett 280:69-72.

Tendolkar I, Ruhrmann S, Brockhaus A, Pukrop R, Klosterkötter J (2002) Remembering or knowing: electrophysiological evidence for an episodic memory deficit in schizophrenia. Psychol Med 32:1261-1271.

Torrey EF (2007) Schizophrenia and the inferior parietal lobule. Schizophr Res 97:215-225.

Vul E, Harris C, Winkielman P, Pashler H (2009) Puzzlingly high correlations of fMRI studies of emotion, personality and social cognition. Perspect Psychol Sci 4:274-290.

Wagner AD, Shannon BJ, Kahn I, Buckner RL (2005) Parietal lobe contributions to episodic memory retrieval. Trends Cogn Sci 9:445-453.

Walla P, Püregger E, Lehrner J, Mayer D, Deecke L, Dal Bianco P (2005) Depth of word processing in Alzheimer patients and normal controls: a magnetoencephalographic (MEG) study. J Neural Transm 112:713-730.

Weiss AP, Goff D, Schacter DL, Ditman T, Freudenreich O, Henderson D,
Heckers S (2006) Fronto-hippocampal function during temporal context monitoring in schizophrenia. Biol Psychiatry 60:1268-1277.

Weiss AP, Goff DC, Roffman JL, Ellis CB, Schacter DL (2007) Neural correlates of source memory performance in schizophrenia. Schizophr Bul 33:388.

Weiss AP, Goff DC, Duff M, Roffman JL, Schacter DL (2008) Distinguishing familiarity-based from source-based memory performance in patients with schizophrenia. Schizophr Res 99:208-217.

Wheeler ME, Buckner RL (2004) Functional-anatomic correlates of remembering and knowing. Neuroimage 21:1337-1349.

Wilding EL (1999) Separating retrieval strategies from retrieval success: an event-related potential study of source monitoring. Neuropsychologia 37:441-454.

Wilding EL, Rugg MD (1996) An event-related potential study of recognition memory with and without retrieval of source. Brain 119: 889-905.

Wolf DH, Gur RC, Valdez JN, Loughead J, Elliott MA, Gur RE, Ragland JD (2007) Alterations of fronto-temporal connectivity during word encoding in schizophrenia. Psychiatry Res 154:221-232. 